\title{
Compact Dual-frequency and Dual-polarized Monopole Antenna
}

\author{
Kang Luo, Bin Chen, YiGang Wang, BoAo Xu, XiaoLi Zhou \\ National Key Laboratory on Electromagnetic Environmental Effects and Electro-optical Engineering \\ PLA University of Science and Technology, China \\ *Luokang89@163.com
}

\begin{abstract}
Keywords: Microstrip antenna; dual band; circularly polarized; linearly polarized
\end{abstract}
\begin{abstract}
In this letter, a compact dual band monopole antenna with circularly polarized (CP) property around $2.4 \mathrm{GHz}$ and linearly polarized (LP) property around $1.57 \mathrm{GHz}$ is presented. The antenna consists of a tree-shaped monopole antenna and a modified grounded plane. To realize CP characteristic, two orthogonal monopole arms (arms A and B) with different lengths are used to generate a phase difference of $90^{\circ}$. Another Y-shaped monopole (arms C and D) connected to the microstrip feed line is loaded to excite the LP mode. Compared with the previous Y-shaped monopole structure, the associated design has a significant improvement (about three times) in the axial ratio (AR) bandwidth and an excellent omnidirectional characteristic in the $\mathrm{H}$-plane at around $1.57 \mathrm{GHz}$.
\end{abstract}

\section{INTRODUCTION}

Nowadays antennas with dual-frequency and dual-polarized characteristics are widely required in wireless services and mobile communication devices. Dual-feed dual-polarized antennas [1-3] and multiple input multiple output (MIMO) [4-5] antennas have been increasingly concerned due to the frequency reuse and polarization diversity. By incorporating the existing systems such as GPS and WLAN systems, an integrated system with improved capacity and complementary coverage can be obtained. However, as the number of embedded antennas increases, it is difficult to achieve high isolation between input ports and low cross polarizations for all ports simultaneously [2-3]. One simple way is using wideband antennas which could cover all frequency bands. However, it may interfere with nearby communication systems. Another solution to this problem is using a multi-functional antenna which is capable of radiating different polarized waves at different frequencies respectively.

In the past, few works describing dual-frequency and dual-polarized antennas with single feed prototype have been presented. A dual-band GPS L1/L2 CP protractor antenna which minimizes the coupling between the two sets of elements is fabricated in [6], while the antenna has a high profile and it is centered on a large copper plate insert for ease of chipping. A multi-band multi-polarization cross spiral antenna for mobile communication devices is presented in [7]. However, the spiral structure makes the antenna relatively complex and the axial radio bandwidth is extremely narrow. A compact antenna with broad bandwidth and quad-sense circular polarization is proposed in [8]. However, the cross-polarization level and main lobes become very worse in the high frequency band.

Turnstile monopole antenna is a good candidate for the applications due to its compact structure and omnidirectional characteristic. Common turnstile antennas typically include two half-wavelength diploes, placed perpendicular to each other and fed by an external coupler network that provides phase difference of $90^{\circ}$ [9]. A modified version of the design is performed to eliminate the coupler network resulting in a single-fed printed Y-shaped monopole antenna having two unequal monopole arms [10], and the AR bandwidth is about $4 \%$ at the center frequency of $2.3 \mathrm{GHz}$.

This letter keeps the compact structure of [10], another Y-shaped monopole connected to the microstrip feed line is loaded to excites the LP mode at around $1.575 \mathrm{GHz}$ [11], and the ground plane is modified to 
enhance the impedance bandwidth. Compared with the previous design [10], the associated antenna has a significant improvement in the AR bandwidth. The radiation pattern of this antenna shows acceptable gain and good omnidirectional performance in the $H$-plane at the low frequency band.

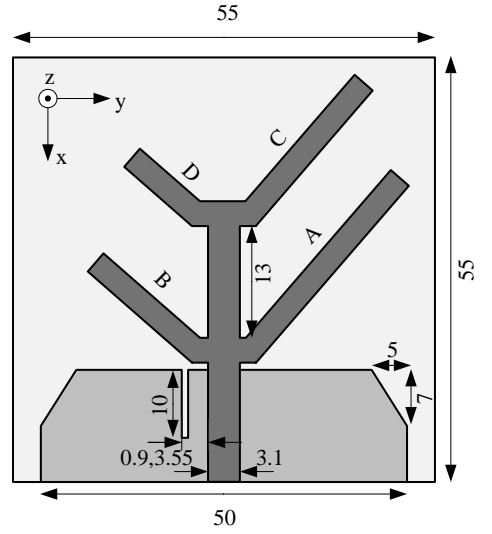

Fig.1 Topology of the proposed antenna

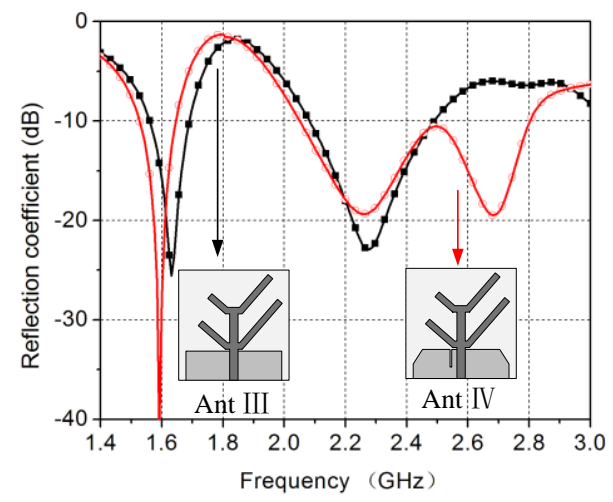

Fig.3 Simulated refection coefficient
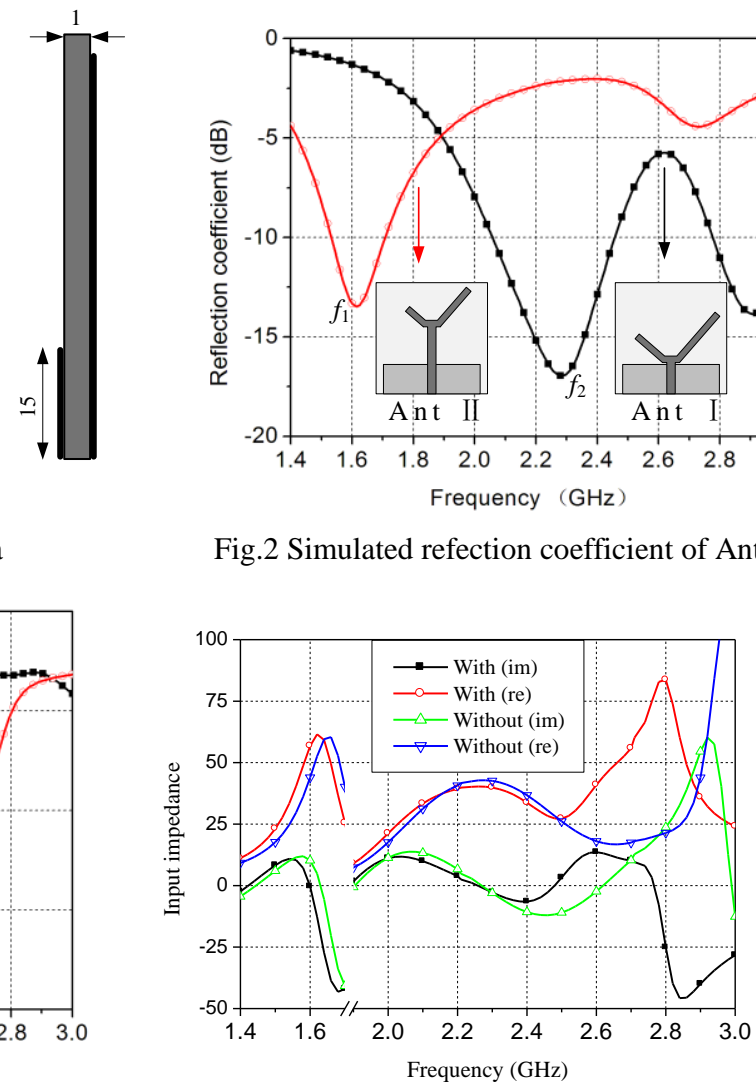

Fig.4 Simulated input impedance $(\Omega)$

\section{ANTENNA DiSEGN}

The base antenna ( Ant I ) consists of a Y-shaped (Dimensions of arms A, B are $31 \mathrm{~mm}, 21 \mathrm{~mm} \mathrm{~A}$ as shown in Fig. 1 ) patch on the top side of the substrate and a rectangular ground plane on the bottom side. The antenna is designed on a square microwave substrate of Rogers 5880 with thickness $1 \mathrm{~mm}$, relative permittivity $\varepsilon_{\mathrm{r}}=2.2$ and dielectric loss tangent $\tan \delta=0.001$. It is fed by a $50 \Omega$ feeding line, where the signal strip has width of $3.1 \mathrm{~mm}$. The overall dimension of the antenna is $55 \times 55 \mathrm{~mm}^{2}$. The optimum performance of the proposed antenna is achieved by the parametric studies carried out by Ansoft HFSS version 12.

Basic antenna ( Ant I ). The design procedure of the proposed antenna is illustrated in Fig. 2 and 3. At first, two orthogonal monopole arms ( arms A and B ) with different lengths are used to generate a phase difference of $90^{\circ}$ at around $2.4 \mathrm{GHz}$ ( Ant I ). Arm A ( $31 \mathrm{~mm}$ ) which is larger than $\lambda / 4(23 \mathrm{~mm})$ produces a wave with relative phase advance, whereas arm B $(21 \mathrm{~mm})$ which is shorter than $\lambda / 4$ produces a wave with relative phase delay. In fact, the bottom portion of the arms is a Tee junction power divider and the arms are two perpendicular linearly polarized elements fed by two signals with equal amplitude. As a result, CP waves are produced when the two arms have equal amplitude and $90^{\circ}$ phase difference. The simulated 3-dB AR bandwidth is about $4 \%$ at the center frequency of $2.4 \mathrm{GHz}$, and the result is similar to that of [10].

The simulated reflection coefficient of the Ant I is presented in Fig. 2. It can be observed that Ant I exhibits two resonant frequencies of $f_{2}$ (around $2.3 \mathrm{GHz}$ ) and $f_{3}$ (around $2.9 \mathrm{GHz}$ ). This is mainly because 
that arm A is much longer than $\lambda / 4$ at $2.4 \mathrm{GHz}$ and broadband 3-dB AR characteristic can be achieved, whereas input impedance can be matched by modifying the ground plane which will be discussed in the next section. If lower AR value is required, arm A should be shorter or arm B should be longer.

Dual polarized antenna ( Ant III ). It is confirmed that the current is mainly concentrated on the $50 \Omega$ feeding line and along the periphery of the patch antenna [12]. Additional resonances to the antenna can be produced by attaching resonant strips of appreciate length to the patch. Consequently, another Y-shaped monopole (Dimensions of arms C, D are $26.5 \mathrm{~mm}, 15 \mathrm{~mm}$ ) connected to the microstrip feed line is loaded to excite the LP mode at around $1.575 \mathrm{GHz}$ ( $f_{1}$, Ant III, Fig. 3 ), and also broaden the AR bandwidth. The performance of Ant III at around $f_{1}$ is similar to that of Ant II as illustrated in Figs. 2 and 3. Both arm C and arm $\mathrm{D}$ are able to control the resonant frequency, and with the increase of their lengths $f_{1}$ shifts downward. Meanwhile, arms C and D affect the CP characteristic ( around $2.4 \mathrm{GHz}$ ) as well. However, the resonant frequencies can be adjusted independently and simply by tuning the corresponding arms. The detail influence will be discussed latter.

Modified antenna ( Ant IV ). The ground plane affects the characteristics of the antenna as well, by properly cutting the two triangle notches and an I-shaped notch on the ground plane, the impedance bandwidth can be much enhanced without significant effect on AR bandwidth. Note that arms A and B slightly shift $f_{1}$ downward and this could be easily compensated by tuning arms $\mathrm{C}$ and $\mathrm{D}$. The topology and detailed optimum geometrical parameters of the modified antenna is shown in Fig.1.

Fig.4 displays the simulated input impedance of the antenna with and without notches. Both of them have the same monopole and ground plane sizes. The sizes and position of the notches provide the impendent matching. For Ant III, the reactance approximate to zero at around $f_{1}$ and $f_{2}$, resulting in the resonance, whereas the resistances are close to zero, leading to the mismatch of the antenna at around $f_{3}$. For Ant IV, the input resistance is greatly improved at around $f_{3}$ and the associated reactance changes from negative to positive value. In general, the antenna without notches exhibits resonances around $1.6 \mathrm{GHz}, 2.3 \mathrm{GHz}$ and $2.9 \mathrm{GHz}$. The modification of the ground plane results in the third resonant frequency shifted from $2.9 \mathrm{GHz}$ to about $2.7 \mathrm{GHz}$, while retaining the original resonances around $1.6 \mathrm{GHz}$ and $2.3 \mathrm{GHz}$. This is mainly because that the I-shaped notch creates an alternate path for the current on the left ground plane, which is no longer balance to that on the right ground plane, rendering the main radiating element within this frequency range. Meanwhile, the AR property is slightly influenced when the size and position of the notches not dramatically change.

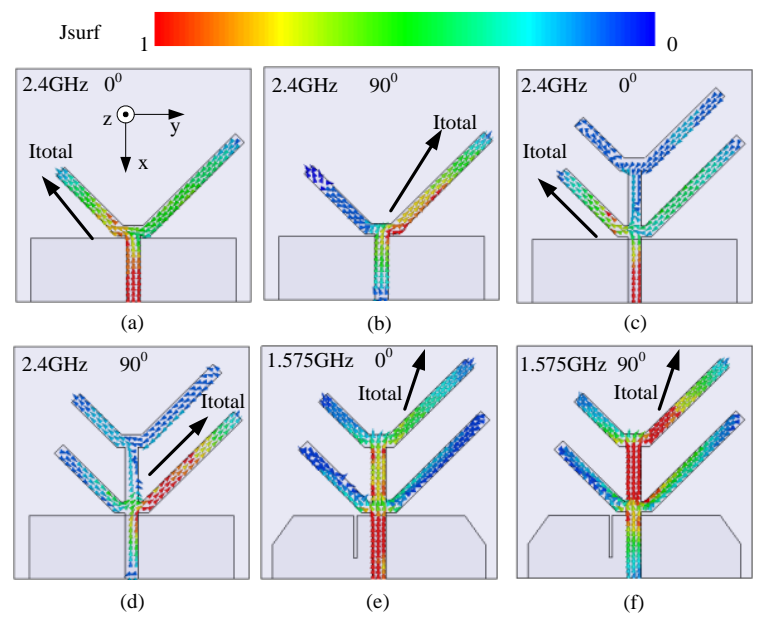

Fig. 5 Simulated surface current distributions of the basic antenna and the proposed antennas

To further analyze the improvement of AR bandwidth and the effect of the ground plane, the surface current distribution of the basic antenna and the proposed antennas with (without) notches on the ground 
plane at $0^{\circ}$ and $90^{\circ}$ have been simulated in Fig. 5. Note that Itotal represents the vector sum of all major current contributions. For the basic antenna at $2.4 \mathrm{GHz}$, the Itotal is oriented in a direction of about $\Phi=-140^{\circ}$ $\left(\Phi=+140^{\circ}\right)$ with respect to the $+x$ axis at $w t=0^{\circ}\left(w t=90^{\circ}\right)$ as shown in Figs.5(a) and (b). The current distribution at $w t=180^{\circ}\left(270^{\circ}\right)$ is just opposite to that at $w t=0^{\circ}\left(90^{\circ}\right)$ and are not plotted in this letter. For the proposed antennas at $2.4 \mathrm{GHz}$, the direction of the currents along arm C (D) at $w t=0^{\circ}\left(90^{\circ}\right)$ is parallel to that of arm B (A) as shown in Figs.5(c) and (d).

Meanwhile, the current density on arm D ( C ) is close to zero, as small current vector with blue in color has been observed. As a result, the Itotal oriented in a direction of about $\Phi=-135^{\circ}\left(\Phi=+135^{\circ}\right)$ with respect to the $+x$. Therefore, lower values of AR and wider AR bandwidth are obtained. For the proposed antennas at $1.575 \mathrm{GHz}$, the currents are mainly distributed along arms $\mathrm{C}$ and the microstrip feed line as shown in Figs.5(e) and (f). The directions of the currents mentioned above are the same (about $\Phi=+165^{\circ}$ with respect to the $+x$ ). This means that arm $\mathrm{C}$ produces linearly polarized wave in the low frequency and also confirms that arms $\mathrm{C}$ and $\mathrm{D}$ are connected as top-loaded.
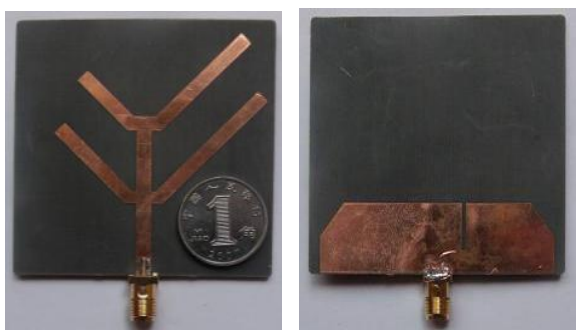

Fig. 6 Photograph of the fabricated antenna

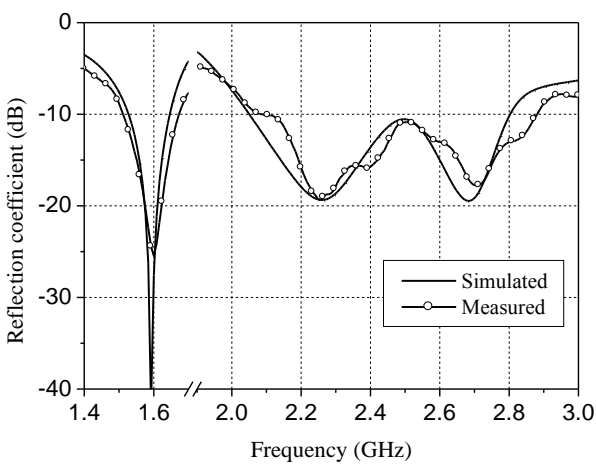

Fig. 7 Simulated and measured refection coefficient

\section{MEASUREMENT RESULTS AND DISCUSSION}

The proposed antenna was fabricated and tested. Fig. 6 shows the prototype of it. From the obtained results shown in Fig. 7, good excitation at around $1.575 \mathrm{GHz}$ and $2.4 \mathrm{GHz}$ are exhibited for the antenna. The measured impedance bandwidth with respect to the center frequency at 1.56 and $2.48 \mathrm{GHz}$, respectively, is $6.53 \%$ and $31.97 \%$. The slight shift is due to the tolerance in manufacturing, simulation error is also contributed to the difference, and this can be improved by setting two "Solution bands" (1.4-1.7 GHz, 1.9-3 $\mathrm{GH})$.

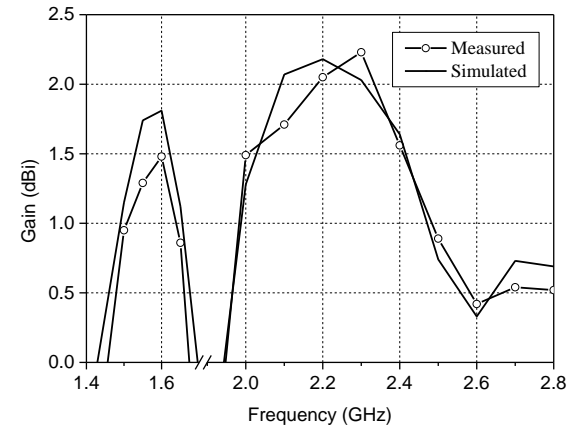

Fig. 8 Simulated and measured gain

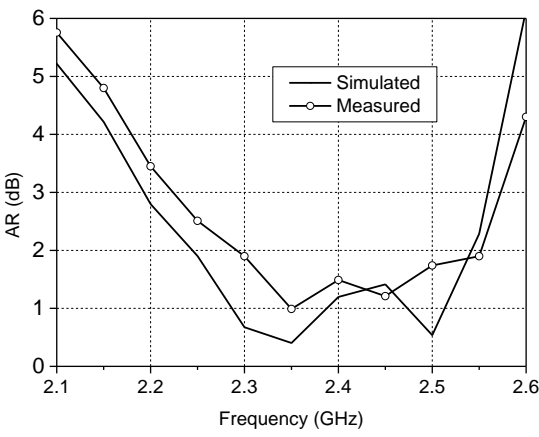

Fig. 9 Simulated and measured AR

The measured gain and AR of the antenna are presented in Fig. 8 and 9, respectively, along with the simulated results. An acceptable gain characteristic is achieved in the input bandwidth, and the peak gain are about $1.49 \mathrm{dBi}(1.6 \mathrm{GHz})$ and $2.06 \mathrm{dBi}(2.2 \mathrm{GHz})$, respectively. Good agreement is obtained between the measured results and simulated results. From $2.22 \mathrm{GHz}$ to $2.56 \mathrm{GHz}$, the measured AR of the antenna is 
below $3 \mathrm{~dB}$ and the $3-\mathrm{dB}$ AR bandwidth is about $14.23 \%$. It can be observed that the measurement results are slightly shifted to the high frequency band, which could be due to fabrication inaccuracies (e.g., overetching) and uncertainties in the substrate effective permittivity.

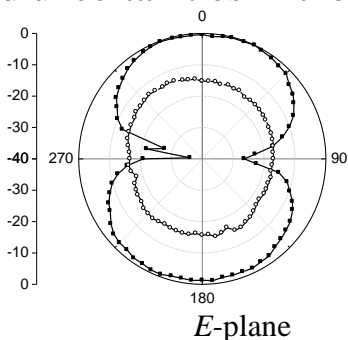

Fig.10 Measured normalized radiation patterns at $1.575 \mathrm{GHz}$

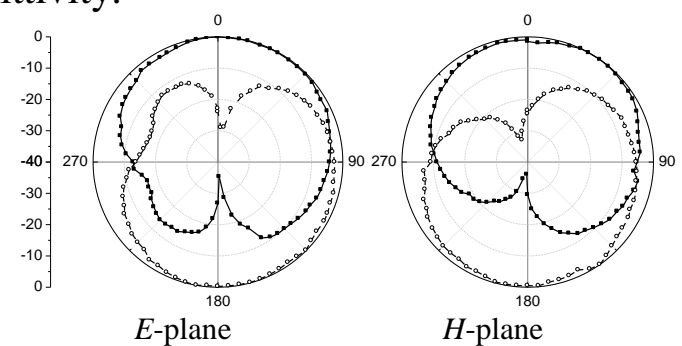

Fig.11 Measured normalized radiation patterns at $2.4 \mathrm{GHz}$ $\longrightarrow$ LHCP $\quad-\sim--$ RHCP

Fig. 10 depicts the measured normalized radiation patterns of the antenna in the $E$-plane and $H$-plane at $1.57 \mathrm{GHz}$. Good broadside radiation patterns with relatively low cross polarizations are observed in the two principal planes. The cross polarization level is about $-15 \mathrm{~dB}$ in the $x-z(E)$ plane and $-14 \mathrm{~dB}$ in the $y-z(H)$ plane. Moreover, the antenna shows omnidirectional radiation patterns in the $H$-plane and dipole-like radiation patterns in the $E$-plane. The cross polarization level is relatively high for angles away from broadside in the low frequency band. Fig. 11 displays the measured normalized right hand circular polarization (RHCP) and left hand circular polarization (LHCP) at $2.4 \mathrm{GHz}$. The radiation pattern is $\mathrm{LHCP}$ for $z>0$ and RHCP for $z<0$. The $3-\mathrm{dB}$ gain beamwidth is about $90^{\circ}$.

\section{CONCLUSION}

A compact dual band microstrip antenna with CP and LP characteristics is investigated. In this model, the AR bandwidth is increased, which is about three times wider than that of the previous similar design [10]. Surface current distributions and input impedance property are presented to discuss the antenna performance. Based on the above analysis, the resonant frequencies can be adjusted independently by tuning the corresponding arms. Measured results show that the proposed antenna has an excellent omnidirectional property in the $H$-plane at around $1.57 \mathrm{GHz}$ and broadband $\mathrm{AR}$ bandwidth at around $2.4 \mathrm{GHz}$.

\section{REFERENCES}

[1] H. Wong, K.-L. Lau, and K.-M. Luk, "Design of dual-polarized L-probe patch antenna arrays with high isolation," IEEE Trans. Antennas and Propag., vol. 52, no. 1, pp. 45-52, Jan. 2004.

[2] K. Luo, W.-P. Ding, Y.-J. Hu, and W.-Q. Cao, "Design of dual-feed dual-polarized microstrip antenna with high isolation and low cross polarization," Progress In Electromagnetics Research Letters, vol. 36, pp. 31-40, Jan. 2013.

[3] C.Y. Chiu, S. Song, R.D. Murch, "Complementary two-port antennas with low mutual coupling," IET Microwaves. Antennas and Propagation. vol. 6, no. 2, pp. 135-141, Jan. 2012.

[4] S.-W. Su, C.-T. Lee, "Low-cost dual-loop-antenna system for dual-WLAN-band access points," IEEE Trans. Antennas and Propag., vol. 59, no. 5, pp. 1652-1659, May. 2011.

[5] S. Zhang, B.-K Lau, A. Sunesson, and S. He, "Closely-Packed UWB MIMO/Diversity Antenna with Different Patterns and Polarizations for USB Dongle Applications," IEEE Trans. Antennas and Propag., vol. 60, n0. 9, pp. 4372-4380, Sep. 2012. 
[6] P. Jin, R. W. Ziolkowski, "Multi-Frequency, Linear and Circular Polarized, Metamaterial-Inspired, Near-Field Resonant Parasitic," IEEE Trans. Antennas and Propag., vol. 59, no. 5, pp. 1446-1459, May. 2011.

[7] Mayumi, Toshiaki, and Bunkyo-cho, "A Multi-Polarization Multi-Band Cross Spiral Antenna for Mobile Communication Devices,” Pro. ISAP, pp.299-302, 2012.

[8] G.-H. Li, H.-Q. Zhai, T. Li, and C.-H. Liang, "A compact antenna with broad bandwidth and quad-sense circular polarization,” IEEE Antennas Wireless Propag. Lett., vol.11, pp. 791-794, 2012.

[9] S. G. M. Darwish, K. F. A. Hussein, and H. A. Mansour, "Circularly polarized cross-dipole turnstile antenna for satellites," in Pro. 21st NRSC, pp. 16-18, Mar. 2004.

[10] A. Ghobadi, M. Dehmollaian, "A printed circularly polarized Y-shaped monopole antenna," IEEE Antennas Wireless Propag. Lett., vol.11, pp. 22-25, 2012.

[11] S.-W. Qu, J.-L. Li, Q. Xue, and C.-H. Chan, "Wideband Periodic Endfire Antenna with Bowtie Dipoles,” IEEE Antennas Wireless Propag. Lett., vol.7, pp. 314-317, 2008.

[12] Nader Behdad, Student Member, IEEE, and Kamal Sarabandi, “A Compact Antenna for UltrawideBand Applications,” IEEE Trans. Antennas and Propag., vol. 53, no. 7, pp. 2185-2192, Jul. 2005. 